\title{
Learning from Chunk-based Feedback in Neural Machine Translation
}

\author{
Pavel Petrushkov and Shahram Khadivi and Evgeny Matusov \\ eBay Inc. \\ Kasernenstr. 25 \\ 52064 Aachen, Germany \\ \{ppetrushkov, skhadivi, ematusov\}@ebay.com
}

\begin{abstract}
We empirically investigate learning from partial feedback in neural machine translation (NMT), when partial feedback is collected by asking users to highlight a correct chunk of a translation. We propose a simple and effective way of utilizing such feedback in NMT training. We demonstrate how the common machine translation problem of domain mismatch between training and deployment can be reduced solely based on chunk-level user feedback. We conduct a series of simulation experiments to test the effectiveness of the proposed method. Our results show that chunk-level feedback outperforms sentence based feedback by up to 2.61\% BLEU absolute.
\end{abstract}

\section{Introduction}

In recent years, machine translation (MT) quality improved rapidly, especially because of advances in neural machine translation (NMT). Most of remaining MT errors arguably come from domain, style, or terminology mismatch between the data on which the MT was trained on and data which it has to translate. It is hard to alleviate this mismatch since usually only limited amounts of relevant training data are available. Yet MT systems deployed on-line in e.g. e-commerce websites or social networks can benefit from user feedback for overcoming this mismatch. Whereas MT users are usually not bilingual, they likely have a good command of the target language and are able to spot severe MT errors in a given translated sentence, sometimes with the help of e.g. an accompanying image, video, or simply prior knowledge.

A common approach to get user feedback for MT is explicit ratings of translations on an n-point
Likert scale. The main problem of such methods is that users are not qualified enough to provide reliable feedback for the whole sentence. Since different users do not adhere to a single set of guidelines, their ratings may be influenced by various factors, such as user expectations, user knowledge, or user satisfaction with the platform. In (Kreutzer et al., 2018), the authors investigate the reliability and validity of real user ratings by re-evaluating five-star ratings by three independent human annotators, however the inter-annotator agreement between experts was relatively low and no correlation to the averaged user rating was found.

Instead of providing a rating, a user might be asked to correct the generated translation, in a process called post-editing. Using corrected sentences for training an NMT system brings larger improvements, but this method requires significant effort and expertise from the user.

Alternatively, feedback can be collected by asking users to mark correct parts (chunks) of the translation (Marie and Max, 2015). It can be seen as the middle ground between quick sentence level rating and more expensive post-editing. We hypothesize that collecting feedback in this form implicitly forces guidelines on the user, making it less susceptible to various user-dependent factors. We expect marking of correct chunks in a translation to be simple enough for non-experts to do quickly and precisely and also be more intuitive than providing a numerical rating.

In this paper, we investigate the empirical hypothesis that NMT is able to learn from the good chunks of a noisy sentence and describe a simple way of utilizing such chunk-level feedback in NMT training. To the best of our knowledge, no dataset with human feedback recorded in this form is available, therefore we experiment with user feedback that was artificially created from parallel data. 
The rest of this paper is structured as follows. In Section 2 we review related work. We describe our partial feedback approach in Section 3. Next we present our experimental results in Section 4, followed by the conclusion in Section 5 .

\section{Related work}

Integrating user ratings in NMT has been studied in (Kreutzer et al., 2017), who view this as a bandit structured prediction task. They demonstrate how the user feedback can be integrated into NMT training and perform a series of experiments using GLEU (Wu et al., 2016) to simulate user feedback. Nguyen et al. (2017) have also studied this problem and adapted an actor-critic approach (Mnih et al., 2016) which has shown to be robust to skewed, high variance feedback from real users.

(Lam et al., 2018) extended the work of (Nguyen et al., 2017) by asking users to provide feedback for partial hypotheses to iteratively generate the translation, their goal is to minimize the required human involvement. They performed simulated experiments using chrF (Popovic, 2015) as simulated feedback.

In all previous works feedback needs to be generated on-line during the training process, however in this paper we focus on the case where there might be a significant time lag between generation of translation and acquiring of the feedback. Lawrence et al. (2017) have proposed a method to leverage user feedback that is available only for logged translated data for a phrase-based statistical machine translation system.

(Kreutzer et al., 2018) have experimented with sentence level star ratings collected from real users of an e-commerce site for logged translation data, but found the feedback to be too noisy to gain improvements. They also proposed using implicit word level task feedback based on query matching in an e-commerce application to improve both translation quality and task specific metrics.

Marie and Max (2015) have proposed an interactive framework which iteratively improves translation generated by the phrase-based system by asking users to select correct parts. Domingo et al. (2016) extended this idea to also include word deletions and substitutions with the goal of reducing human effort in translation.

Grangier and Auli (2017) have studied the task of paraphrasing an already generated translation by excluding words that the user has marked as incorrect. They modify NMT model to also accept the marked target sentence as input and train it to produce similar sentences that do not contain marked words.

(Chen et al., 2017; Wang et al., 2017) have proposed sentence level weighting method for domain adaptation in NMT.

\section{Method}

In this work we use the encoder-decoder NMT architecture with attention, proposed by (Bahdanau et al., 2014; Sutskever et al., 2014). NMT model is trained to maximize the conditional likelihood of a target sentence $e_{1}^{I}: e_{1}, \ldots, e_{I}$ given a source sentence $f_{1}^{J}: f_{1}, \ldots, f_{J}$ from a parallel dataset $D$ :

$$
\mathcal{L}=\sum_{f_{1}^{J}, e_{1}^{I} \in D} \sum_{i=1}^{I} \log p\left(e_{i} \mid e_{1}^{i-1}, f_{1}^{J}\right) .
$$

Training objective (1) is appropriate when the target sentence $e_{1}^{I}$ comes from real data. However, we would like to benefit from model-generated sentences $\tilde{e}_{1}^{I}$ by introducing partial feedback.

We assume that partial feedback for a sentence $\tilde{e}_{1}^{I}$ is given as a sequence of binary values $w_{1}^{I}$ : $w_{1}, \ldots, w_{I}$, such that $w_{i}=1$ if the word $\tilde{e}_{i}$ is marked as correct, $w_{i}=0$ if it is unrated or incorrect. We propose a simple modification to the loss in Equation (1):

$$
\mathcal{L}_{P F}=\sum_{f_{1}^{J}, \tilde{e}_{1}^{I}, w_{1}^{I} \in D} \sum_{i=1}^{I} w_{i} \log p\left(\tilde{e}_{i} \mid \tilde{e}_{1}^{i-1}, f_{1}^{J}\right)
$$

Considering the definition of the binary partial feedback, the model would be trained to predict correct target words, while ignoring unrated and incorrect ones. However, incorrect words are still used as inputs to the model and influence the prediction context of correct words.

While partial feedback is gathered in a binary form (selected/not selected), word weights $w_{i}$ can take any real value, depending on the weight assignment scheme.

Our training objective can be seen as a generalization of sentence level weighting method (Chen et al., 2017; Wang et al., 2017). The special case of sentence level weight can be expressed as $w_{i}=$ $w, \forall i$, where $w$ is the weight for sentence $\tilde{e}_{1}^{I}$. 
We differentiate between two practical methods of obtaining the partial feedback data. First, gathering the feedback from humans, by presenting them with translations and asking to highlight correct words. This method is expected to produce high quality feedback, but is relatively expensive and, to the best of our knowledge, no such dataset is publicly available.

Another method is to generate partial feedback automatically using heuristics or statistical models. This type of feedback would be cheap to obtain, but is unlikely to be of high quality.

In this paper, to show the effectiveness of high quality chunk feedback, we generate artificial feedback by comparing model predictions to reference translations using heuristic methods. This approach is cheap, produces high quality feedback, but is not practically useful, because it requires access to reference human translation.

We have experimented with several methods of extracting artificial feedback. A simple matching method assigns $w_{i}=1$ if predicted word $\tilde{e}_{i}$ is present in reference translation at any position, and $w_{i}=0$ otherwise. A slightly more sophisticated method is to find the longest common substring (LCS) between the predicted and reference translations and set the weights for words which belong to the LCS to 1 , and to 0 otherwise. In our experiments we have found the latter method to perform slightly better.

\section{Experiments}

In this section, we conduct a series of experiments to study how well an NMT system is able to learn only from partial user feedback when this feedback is given for in-domain translations, whereas the baseline system is trained on out-of-domain data.

\subsection{Datasets}

We report results on two datasets: WMT 2017 German to English news translation task (Bojar et al., 2017) and an in-house English to Spanish dataset in the e-commerce domain. On all data we apply byte-pair encoding (Sennrich et al., 2016) with 40,000 merge operations learned separately for each language.

For each dataset we separate the larger outof-domain and smaller in-domain training data. For De-En we use $1.8 \mathrm{M}$ sentence pairs randomly sampled from available parallel corpora as out- of-domain data and $800 \mathrm{~K}$ sentence pairs sampled from back-translated monolingual and unused parallel corpora as in-domain data. For En-Es we have $2.7 \mathrm{M}$ out-of-domain and $1.5 \mathrm{M}$ in-domain sentence pairs. We evaluate our models on newstest2016 (2999 sentence pairs) for the De-En task and an in-house test set of 1000 sentence pairs for the En-Es task using case-insensitive BLEU (Papineni et al., 2002) and TER (Snover et al., 2006).

We have implemented our NMT model using TensorFlow (Abadi et al., 2015) library. Our encoder is a bidirectional LSTM with a layer size of 512; our decoder is an LSTM with 2 layers of the same size. We also use embedding size of 512 and MLP attention layer. We train our networks using SGD with a learning rate schedule that starts gradually decaying to 0.01 after the initial 4 epochs. As regularization we use dropout on the RNN inputs with dropping probability of 0.2 .

\subsection{Results}

We pre-train baseline NMT models on parallel out-of-domain data for 15 epochs. We then use the pre-trained model to generate translations from the source side of parallel in-domain corpus. Using heuristics described in Section 3 and the reference target side of the in-domain corpus we generate artificial partial feedback to simulate real user input. Then we continue training with a small learning rate for another 10 epochs on in-domain data with or without user feedback.

In Table 1, we show the effect of different types of feedback on translation performance. First, we see that even using no feedback slightly improves the model due to self-training on automatically translated in-domain data.

Introducing sentence level feedback improves De-En and En-Es models by at most $0.2 \%$ and $0.6 \%$ absolute BLEU, respectively. Sentence level feedback is artificially generated from parallel corpora using heuristics, similar to the ones described in Section 3, but $w_{i}, \forall i$ are set to the same sentence weight $w$. For example, we have tried using sentence BLEU (sBLEU) and a binary rule, which outputs 1 if more than $33 \%$ of predicted words were marked as correct, and 0 otherwise (binary). We have also experimented with other heuristics, but did not achieve better results.

Finally, chunk-based feedback approach based on LCS improves on top of sentence level feedback by another $0.7 \%$ and $2.6 \%$ BLEU for De- 


\begin{tabular}{lcc|cc}
\hline & \multicolumn{2}{c}{ De-En } & \multicolumn{2}{c}{ En-Es } \\
\cline { 2 - 5 } & BLEU & TER & BLEU & TER \\
& {$[\%]$} & {$[\%]$} & {$[\%]$} & {$[\%]$} \\
\hline Baseline & 30.6 & 49.6 & 32.7 & 52.6 \\
+self-training & 31.4 & 48.1 & 35.6 & 49.1 \\
+sent-sBLEU & 31.4 & 48.1 & 36.0 & 48.4 \\
+ sent-binary & 31.6 & 47.8 & 36.2 & 47.6 \\
+chunk-match & 32.2 & 47.0 & 37.9 & 45.4 \\
+chunk-lcs & $\mathbf{3 2 . 3}$ & $\mathbf{4 6 . 5}$ & $\mathbf{3 8 . 8}$ & $\mathbf{4 4 . 5}$ \\
\hline
\end{tabular}

Table 1: Chunk-level feedback compared to sentence-level feedback. Self-training is equivalent to having no feedback or setting all $w_{i}=1, \forall i$ in the training objective in Eq. (2). sent-sBLEU and sent-binary are sentence-level methods with sentence BLEU and binary weighting rules, defined as in Section 4.2. chunk-match and chunklcs-level feedback refers to assigning $w_{i}$ using simple matching or LCS method described in Section 3 .

En and En-Es, respectively. We also note a significant improvement of $1.3 \%$ and $3.1 \%$ in TER. Chunk-based approach based on simple matching also outperforms sentence level methods, but not by as much as lcs-based, which suggests that this method benefits more from consecutive segments, rather than single correct words.

We believe that the success of the partial feedback approach can be explained by the fact that often a sentence can be split into chunks which can be translated independently of the context. Reinforcement of the correct translation of such a chunk in one training example seems to positively affect translations of such chunks in other, different sentences. By focusing on the good and masking out erroneous chunks, partial feedback acts as a precise noise reduction method.

We have also trained the models using finetuning (Luong and Manning, 2015) on the reference target in-domain data, which further improved translation by $2 \%$ and $3.8 \%$ BLEU on De-En and En-Es compared to using chunk-based feedback. We note that by using partial feedback we are able to recover between $30 \%$ and $45 \%$ of improvements that come from in-domain adaptation.

\subsection{Robustness}

The proposed artificially generated partial feedback is very precise as it does not introduce any

\begin{tabular}{|c|c|c|c|c|c|}
\hline \multirow[b]{2}{*}{ \# } & & \multicolumn{2}{|c|}{ De-En } & \multicolumn{2}{|c|}{ En-Es } \\
\hline & & $\begin{array}{c}\text { BLEU } \\
{[\%]}\end{array}$ & $\begin{array}{l}\text { TER } \\
{[\%]}\end{array}$ & $\begin{array}{c}\text { BLEU } \\
{[\%]}\end{array}$ & $\begin{array}{l}\text { TER } \\
{[\%]}\end{array}$ \\
\hline 1 & $\begin{array}{l}\text { Chunk-level } \\
\text { feedback }\end{array}$ & 32.3 & 46.5 & 38.8 & 44.5 \\
\hline \multicolumn{6}{|c|}{ Under selection ratio: } \\
\hline 2 & $25 \%$ & 32.2 & 47.0 & 38.9 & 45.0 \\
\hline 3 & $50 \%$ & 31.9 & 47.4 & 38.1 & 45.6 \\
\hline 4 & $75 \%$ & 31.4 & 47.9 & 36.7 & 46.7 \\
\hline \multicolumn{6}{|c|}{ Incorrect selection ratio: } \\
\hline 5 & $10 \%$ & 32.0 & 47.2 & 38.1 & 44.9 \\
\hline 6 & $25 \%$ & 31.5 & 47.9 & 37.2 & 46.9 \\
\hline 7 & $50 \%$ & 30.9 & 48.8 & 35.6 & 50.0 \\
\hline 8 & $\# 2+\# 5$ & 31.6 & 47.7 & 38.1 & 45.5 \\
\hline
\end{tabular}

Table 2: Impact of user errors on the translation performance. Under selection ratio\% indicates on average what percentage of words in a correct chunk have not been selected in user simulation, but all selected words are correct. Incorrect selection ratio\% indicates what percentage of words are incorrectly selected, here the total number of marked words is the same as in chunk-level feedback. In the last row, $10 \%$ of marked words are actually incorrect and the total number of marked words is $25 \%$ less compared to system in row 1 .

type of noise in marking of good chunks. For example, on the En-Es dataset artificial methods mark $40 \%$ of all words as correct. However, a user might not mark all the correct words in a sentence, but select only a few.

Furthermore, artificially generated partial feedback does not contain noise, given that the reference translation is adequate. However, users may make mistakes in selection. We differentiate two types of errors that a user can make: under selection, when a correct word was not marked; and incorrect selection, when an incorrect word was marked as correct.

To anticipate the impact of these mistakes we experiment with deliberately impairing the feedback in Table 2 . We see that randomly dropping $25 \%$ of the selection has very little effect on the model, while dropping 50\% and more decreases the translation performance significantly, yet still performing at the same level or better than selftraining system.

When selection contains noise, the impact already becomes noticeable at $10 \%$. Increasing the amount of noise up to $25 \%$ decreases the perfor- 
mance by $1.6 \%$ BLEU in En-Es task. At $50 \%$ noise level, which is similar to random selection, there is no improvement from using feedback at all. While we expect users to provide mostly clean feedback, this result indicates the necessity of cleaning user feedback data, e.g. by aggregating feedback from multiple users.

We have also experimented with replacing unselected words by random noise and saw only small decrease in translation performance, which suggests that our approach is able to benefit from very poor translations, as long as the selected chunk is correct.

\subsection{Example}

An example where the NMT system with chunkbased feedback yields a better translation in comparison to other systems is the German sentence "Die Krise ist vorüber." ("The crisis is over."). The German word "vorüber" is rare and ambiguous, especially after the BPE-based splitting. The system with self-training translates the sentence as "The crisis is above all.", whereas the system with chunk-based feedback exactly matches the reference translation. We have analyzed the feedback training set: in that data, out of nine occurrences of the word "vorüber" with the reference translation "over", the baseline system got it right three times, getting rewards for the chunks "is over ...", "is over", "is over."

\section{Conclusion and future work}

In this work, we have proposed a simple way to integrate partial chunk-based feedback into NMT training. We have experimented with artificially created partial feedback and shown that using partial feedback results in significant improvements of MT quality in terms of BLEU and TER. We have shown that chunk-level feedback can be used more effectively than sentence-level feedback. We have studied the robustness of our approach and observed that our model is robust against a moderate amount of noise.

We argue that collecting partial feedback by asking users to highlight correct parts of a translation is more intuitive for users than sentence level ratings and leads to less variation and errors.

In the future, we plan to investigate how to integrate negative partial user feedback, as well as automatic feedback generation methods which do not rely on existing parallel data.

\section{References}

Martín Abadi, Ashish Agarwal, Paul Barham, Eugene Brevdo, Zhifeng Chen, Craig Citro, Greg S. Corrado, Andy Davis, Jeffrey Dean, Matthieu Devin, Sanjay Ghemawat, Ian Goodfellow, Andrew Harp, Geoffrey Irving, Michael Isard, Yangqing Jia, Rafal Jozefowicz, Lukasz Kaiser, Manjunath Kudlur, Josh Levenberg, Dan Mané, Rajat Monga, Sherry Moore, Derek Murray, Chris Olah, Mike Schuster, Jonathon Shlens, Benoit Steiner, Ilya Sutskever, Kunal Talwar, Paul Tucker, Vincent Vanhoucke, Vijay Vasudevan, Fernanda Viégas, Oriol Vinyals, Pete Warden, Martin Wattenberg, Martin Wicke, Yuan Yu, and Xiaoqiang Zheng. 2015. TensorFlow: Large-scale machine learning on heterogeneous systems. Software available from tensorflow.org.

Dzmitry Bahdanau, Kyunghyun Cho, and Yoshua Bengio. 2014. Neural machine translation by jointly learning to align and translate. CoRR, abs/1409.0473.

Ondřej Bojar, Rajen Chatterjee, Christian Federmann, Yvette Graham, Barry Haddow, Shujian Huang, Matthias Huck, Philipp Koehn, Qun Liu, Varvara Logacheva, Christof Monz, Matteo Negri, Matt Post, Raphael Rubino, Lucia Specia, and Marco Turchi. 2017. Findings of the 2017 conference on machine translation (wmt17). In Proceedings of the Second Conference on Machine Translation, Volume 2: Shared Task Papers, pages 169-214, Copenhagen, Denmark. Association for Computational Linguistics.

Boxing Chen, Colin Cherry, George Foster, and Samuel Larkin. 2017. Cost weighting for neural machine translation domain adaptation. In Proceedings of the First Workshop on Neural Machine Translation, pages 40-46. Association for Computational Linguistics.

Miguel Domingo, Alvaro Peris, and Francisco Casacuberta. 2016. Interactive-predictive translation based on multiple word-segments. In Proceedings of the 19th Annual Conference of the European Association for Machine Translation, pages 282-291.

David Grangier and Michael Auli. 2017. Quickedit: Editing text \& translations via simple delete actions. CoRR, abs/1711.04805.

Julia Kreutzer, Shahram Khadivi, Evgeny Matusov, and Stefan Riezler. 2018. Can neural machine translation be improved with user feedback? CoRR, abs/1804.05958.

Julia Kreutzer, Artem Sokolov, and Stefan Riezler. 2017. Bandit structured prediction for neural sequence-to-sequence learning. In Proceedings of the 55th Annual Meeting of the Association for Computational Linguistics, ACL 2017, Vancouver, Canada, July 30 - August 4, Volume 1: Long Papers, pages 1503-1513. 
Tsz Kin Lam, Julia Kreutzer, and Stefan Riezler. 2018. A Reinforcement Learning Approach to Interactive-Predictive Neural Machine Translation. ArXiv:1805.01553v1.

Carolin Lawrence, Artem Sokolov, and Stefan Riezler. 2017. Counterfactual learning from bandit feedback under deterministic logging : A case study in statistical machine translation. In Proceedings of the 2017 Conference on Empirical Methods in Natural Language Processing, EMNLP 2017, Copenhagen, Denmark, September 9-11, 2017, pages 2566-2576.

Minh-Thang Luong and Christopher D. Manning. 2015. Stanford neural machine translation systems for spoken language domains. In Proceedings of the International Workshop on Spoken Language Translation : December 3-4, 2015, Da Nang, Vietnam / Edited by Marcello Federico, Sebastian Stker, Jan Niehues.

Benjamin Marie and Aurélien Max. 2015. Touchbased pre-post-editing of machine translation output. In Proceedings of the 2015 Conference on Empirical Methods in Natural Language Processing, pages 1040-1045, Lisbon, Portugal. Association for Computational Linguistics.

Volodymyr Mnih, Adria Puigdomenech Badia, Mehdi Mirza, Alex Graves, Timothy Lillicrap, Tim Harley, David Silver, and Koray Kavukcuoglu. 2016. Asynchronous methods for deep reinforcement learning. In Proceedings of The 33rd International Conference on Machine Learning, volume 48 of Proceedings of Machine Learning Research, pages 19281937, New York, New York, USA. PMLR.

Khanh Nguyen, Hal Daumé III, and Jordan L. BoydGraber. 2017. Reinforcement learning for bandit neural machine translation with simulated human feedback. In Proceedings of the 2017 Conference on Empirical Methods in Natural Language Processing, EMNLP 2017, Copenhagen, Denmark, September 9-11, 2017, pages 1464-1474.

Kishore Papineni, Salim Roukos, Todd Ward, and WeiJing Zhu. 2002. Bleu: A method for automatic evaluation of machine translation. In Proceedings of the 40th Annual Meeting on Association for Computational Linguistics, ACL '02, pages 311-318, Stroudsburg, PA, USA. Association for Computational Linguistics.

Maja Popovic. 2015. chrf: character n-gram f-score for automatic MT evaluation. In Proceedings of the Tenth Workshop on Statistical Machine Translation, WMT@EMNLP 2015, 17-18 September 2015, Lisbon, Portugal, pages 392-395. The Association for Computer Linguistics.

Rico Sennrich, Barry Haddow, and Alexandra Birch. 2016. Neural machine translation of rare words with subword units. In Proceedings of the 54th Annual Meeting of the Association for Computational Linguistics, ACL 2016, August 7-12, 2016, Berlin, Germany, Volume 1: Long Papers.
Matthew Snover, Bonnie Dorr, Richard Schwartz, Linnea Micciulla, and John Makhoul. 2006. A study of translation edit rate with targeted human annotation. In In Proceedings of Association for Machine Translation in the Americas, pages 223-231.

Ilya Sutskever, Oriol Vinyals, and Quoc V. Le. 2014. Sequence to sequence learning with neural networks. In Proceedings of the 27th International Conference on Neural Information Processing Systems - Volume 2, NIPS'14, pages 3104-3112, Cambridge, MA, USA. MIT Press.

Rui Wang, Masao Utiyama, Lemao Liu, Kehai Chen, and Eiichiro Sumita. 2017. Instance weighting for neural machine translation domain adaptation. In Proceedings of the 2017 Conference on Empirical Methods in Natural Language Processing, pages 1482-1488. Association for Computational Linguistics.

Yonghui Wu, Mike Schuster, Zhifeng Chen, Quoc V. Le, Mohammad Norouzi, Wolfgang Macherey, Maxim Krikun, Yuan Cao, Qin Gao, Klaus Macherey, Jeff Klingner, Apurva Shah, Melvin Johnson, Xiaobing Liu, ukasz Kaiser, Stephan Gouws, Yoshikiyo Kato, Taku Kudo, Hideto Kazawa, Keith Stevens, George Kurian, Nishant Patil, Wei Wang, Cliff Young, Jason Smith, Jason Riesa, Alex Rudnick, Oriol Vinyals, Greg Corrado, Macduff Hughes, and Jeffrey Dean. 2016. Google's neural machine translation system: Bridging the gap between human and machine translation. CoRR, abs/1609.08144. 